\title{
ORBIT EQUIVALENT SUBSTITUTION DYNAMICAL SYSTEMS AND COMPLEXITY
}

\author{
S. BEZUGLYI AND O. KARPEL
}

(Communicated by Bryna Kra)

\begin{abstract}
For any primitive proper substitution $\sigma$, we give explicit constructions of countably many pairwise non-isomorphic substitution dynamical systems $\left\{\left(X_{\zeta_{n}}, T_{\zeta_{n}}\right)\right\}_{n=1}^{\infty}$ such that they all are (strong) orbit equivalent to $\left(X_{\sigma}, T_{\sigma}\right)$. We show that the complexity of the substitution dynamical systems $\left\{\left(X_{\zeta_{n}}, T_{\zeta_{n}}\right)\right\}$ is the essential difference that prevents them from being isomorphic. Given a primitive (not necessarily proper) substitution $\tau$, we find a stationary simple properly ordered Bratteli diagram with the least possible number of vertices such that the corresponding Bratteli-Vershik system is orbit equivalent to $\left(X_{\tau}, T_{\tau}\right)$.
\end{abstract}

\section{INTRODUCTION}

The seminal paper 11 answered, among other outstanding results, the question of orbit equivalence of uniquely ergodic minimal homeomorphisms of a Cantor set. It was proved that two such minimal systems, $(X, T)$ and $(Y, S)$, are orbit equivalent if and only if the clopen values sets $S(\mu)=\{\mu(E): E$ clopen in $X\}$ and $S(\nu)=\{\nu(F): F$ clopen in $Y\}$ coincide, where $\mu$ and $\nu$ are the unique invariant measures with respect to $T$ and $S$, respectively. It is well known now that Bratteli diagrams play an extremely important role in the study of homeomorphisms of Cantor sets because any minimal (and even aperiodic) homeomorphism of a Cantor set is conjugate to the Vershik map acting on the path space of a Bratteli diagram [11], 12], 14]. This realization turns out to be useful in many cases, in particular for the study of substitution dynamical systems, because the corresponding Bratteli diagrams are of the simplest form. It was proved in [7] that the class of minimal substitution dynamical systems coincides with Bratteli-Vershik systems of stationary simple Bratteli diagrams. Later on, it was shown in 3 that a similar result is true for aperiodic dynamical systems. These facts allow us to easily find the clopen values set $S(\mu)$ for a substitution dynamical system in terms of the matrix of substitution (see [4] and subsection 2.2). In order to construct a minimal substitution dynamical system which is orbit equivalent to a given one, $\left(X_{\sigma}, T_{\sigma}\right)$ (in other words, a simple stationary Bratteli diagram $\left.B_{\sigma}\right)$, one has to find another stationary simple Bratteli diagram $B$ such that the clopen values set $S(\mu)$ is kept unchanged, where $\mu$ is a unique $T_{\sigma}$-invariant measure. Moreover, if one wants to have a substitution dynamical system which is strongly orbit equivalent to $\left(X_{\sigma}, T_{\sigma}\right)$, then additionally the dimension group of the diagram $B_{\sigma}$ must be unchanged. Of course, we are not

Received by the editors January 10, 2012 and, in revised form, September 18, 2012 and January 3, 2013.

2010 Mathematics Subject Classification. Primary 37B10; Secondary 37A20, 37B05. 
interested in the case when powers of $\sigma$ are considered since it leads trivially to conjugate substitution systems.

We focus here on the study of (strong) orbit equivalence of minimal substitution dynamical systems. Aperiodic non-minimal substitution systems were considered before in [2]. There were built countably many substitution dynamical systems such that their full ergodic invariant measures were homeomorphic (and hence the measures had the same clopen values sets $S(\mu)$ ) but the systems were pairwise not orbit equivalent. The non-minimality of the systems was essential for the paper [2]. We note that the simplest case for minimal systems when the invariant measure $\mu$ has rational $S(\mu)$ and $\lambda$ is an integer that was studied in [17. There were found numerical complete invariants for topological orbit equivalence in such a class of systems and the assumption of rationality of $S(\mu)$ was important for the proofs.

The main results of the present paper are as follows. Let $\left(X_{\sigma}, T_{\sigma}\right)$ be a minimal substitution dynamical system and let $B_{\sigma}$ be a stationary simple Bratteli diagram corresponding to $\left(X_{\sigma}, T_{\sigma}\right)$. We give an explicit construction of countably many substitutions $\left\{\zeta_{n}\right\}_{n=1}^{\infty}$ defined on the Bratteli diagrams $\left\{B_{n}\right\}_{n=1}^{\infty}$, obtained by telescoping $B_{\sigma}$, such that the systems $\left\{\left(X_{\zeta_{n}}, T_{\zeta_{n}}\right)\right\}_{n=1}^{\infty}$ are strong orbit equivalent to $\left(X_{\sigma}, T_{\sigma}\right)$ and pairwise non-isomorphic. In the other construction, we build pairwise non-isomorphic orbit equivalent minimal substitution dynamical systems by using alphabets of different cardinality.

In both constructions we use the complexity function $n \mapsto p_{\sigma}(n)$ to distinguish non-isomorphic systems. Recall that the function $p_{\sigma}(n)$ counts the number of words of length $n$ in the infinite sequence invariant with respect to $\sigma$. In the first construction, the incidence matrices of built substitution systems are the powers of the incidence matrix of the substitution system $\left(X_{\sigma}, T_{\sigma}\right)$. In the case of a fixed alphabet, the complexity function can be made to increase by enlarging the length of substitution and an appropriate permutation of letters. Using this method, we produce a countable family of pairwise non-isomorphic strong orbit equivalent substitution systems. In the second construction, the complexity of the systems is forced to grow by increasing the number of letters in the alphabet. In other words, for a proper substitution $\sigma$ defined on the alphabet $\mathcal{A}$, we find countably many proper substitutions $\left\{\zeta_{n}\right\}_{n=1}^{\infty}$ on alphabets $\mathcal{A}_{n}$ of different cardinality such that $\left(X_{\sigma}, T_{\sigma}\right)$ is orbit equivalent to $\left(X_{\zeta_{n}}, T_{\zeta_{n}}\right)$, but the set $\left\{\left(X_{\zeta_{n}}, T_{\zeta_{n}}\right)\right\}_{n=1}^{\infty}$ consists of pairwise non-isomorphic substitution dynamical systems.

Given the Bratteli-Vershik system on a simple stationary diagram $(B, \leq)$, we find an orbit equivalent stationary Bratteli-Vershik system with the least possible number of vertices. This number is the degree of the algebraic integer $\lambda$, the Perron-Frobenius eigenvalue of the matrix transpose to the incidence matrix of $B$.

\section{Preliminaries}

2.1. Minimal Cantor systems. A minimal Cantor system is a pair $(X, T)$ where $X$ is a Cantor space and $T: X \rightarrow X$ is a minimal homeomorphism; i.e. for every $x \in X$ the set $\operatorname{Orb}_{T}(x)=\left\{T^{n}(x) \mid n \in \mathbb{Z}\right\}$ is dense in $X$.

Given a minimal Cantor system $(X, T)$ and a clopen $A \subset X$, let $r_{A}(x)=\min \{n \geq$ $\left.1: T^{n}(x) \in A\right\}$ be a continuous integer-valued map defined on $A$. Then $T_{A}(x)=$ $T^{r_{A}}(x)$ is a homeomorphism of $A$, and $\left(A, T_{A}\right)$ is a Cantor minimal system and is said to be induced from $(X, T)$.

There are several notions of equivalence for minimal Cantor systems. 
Definition 2.1. Let $(X, T)$ and $(Y, S)$ be two minimal Cantor systems. Then:

(1) $(X, T)$ and $(Y, S)$ are conjugate (or isomorphic) if there exists a homeomorphism $h: X \rightarrow Y$ such that $h \circ T=S \circ h$.

(2) $(X, T)$ and $(Y, S)$ are orbit equivalent if there exists a homeomorphism $h: X \rightarrow Y$ such that $h\left(\operatorname{Orb}_{T}(x)\right)=\operatorname{Orb}_{S}(h(x))$ for every $x \in X$. In other words, there exist functions $n, m: X \rightarrow \mathbb{Z}$ such that for all $x \in X, h \circ T(x)=S^{n(x)} \circ h(x)$ and $h \circ T^{m(x)}=S \circ h(x)$. The functions $n, m$ are called the orbit cocycles associated to $h$.

(3) $(X, T)$ and $(Y, S)$ are strong orbit equivalent if they are orbit equivalent and each of the corresponding orbit cocycles has at most one point of discontinuity.

(4) $(X, T)$ and $(Y, S)$ are Kakutani equivalent if they both have clopen subsets such that the corresponding induced systems are conjugate.

(5) $(X, T)$ and $(Y, S)$ are Kakutani orbit equivalent if they both have clopen subsets such that the corresponding induced systems are orbit equivalent.

\subsection{Bratteli diagrams.}

Definition 2.2. A Bratteli diagram is an infinite graph $B=(V, E)$ such that the vertex set $V=\bigcup_{i \geq 0} V_{i}$ and the edge set $E=\bigcup_{i \geq 1} E_{i}$ are partitioned into disjoint subsets $V_{i}$ and $E_{i}$ such that:

(i) $V_{0}=\left\{v_{0}\right\}$ is a single point;

(ii) $V_{i}$ and $E_{i}$ are finite sets;

(iii) there exist a range map $r$ and a source map $s$ from $E$ to $V$ such that $r\left(E_{i}\right)=V_{i}, s\left(E_{i}\right)=V_{i-1}$, and $s^{-1}(v) \neq \emptyset, r^{-1}\left(v^{\prime}\right) \neq \emptyset$ for all $v \in V$ and $v^{\prime} \in V \backslash V_{0}$.

The pair $\left(V_{i}, E_{i}\right)$ or just $V_{i}$ is called the $i$-th level of the diagram $B$. A sequence of edges $\left(e_{i}: e_{i} \in E_{i}\right)$ such that $r\left(e_{i}\right)=s\left(e_{i+1}\right)$ is called a path. We denote by $X_{B}$ the set of all infinite paths starting at the vertex $v_{0}$. This set is endowed with the standard topology turning $X_{B}$ into a Cantor set.

Given a Bratteli diagram $B=(V, E)$, we define a sequence of incidence matrices $F_{n}=\left(f_{v w}^{(n)}\right)$ of $B: f_{v w}^{(n)}=\left|\left\{e \in E_{n+1}: r(e)=v, s(e)=w\right\}\right|$, where $v \in V_{n+1}$ and $w \in V_{n}$. Here and thereafter $|V|$ denotes the cardinality of the set $V$. A Bratteli diagram is called stationary if $F_{n}=F_{1}$ for every $n \geq 2$.

A Bratteli diagram $B^{\prime}=\left(V^{\prime}, E^{\prime}\right)$ is called the telescoping of a Bratteli diagram $B=(V, E)$ to a sequence $0=m_{0}<m_{1}<\ldots$ if $V_{n}^{\prime}=V_{m_{n}}$ and $E_{n}^{\prime}$ is the set of all paths from $V_{m_{n-1}}$ to $V_{m_{n}}$, i.e. $E_{n}^{\prime}=E_{m_{n-1}} \circ \ldots \circ E_{m_{n}}=\left\{\left(e_{m_{n-1}}, \ldots, e_{m_{n}}\right): e_{i} \in\right.$ $\left.E_{i}, r\left(e_{i}\right)=s\left(e_{i+1}\right)\right\}$.

Observe that every vertex $v \in V$ is connected to $v_{0}$ by a finite path, and the set $E\left(v_{0}, v\right)$ of all such paths is finite. A Bratteli diagram is called simple if for any $n>0$ there exists $m>n$ such that any two vertices $v \in V_{n}$ and $w \in V_{m}$ are connected by a finite path.

A Bratteli diagram $B=(V, E)$ is called ordered if every set $r^{-1}(v), v \in \bigcup_{n>1} V_{n}$, is linearly ordered. Given an ordered Bratteli diagram $(B, \leq)=(V, E, \leq)$, any two paths from $E\left(v_{0}, v\right)$ are comparable with respect to the lexicographical order [12. We call a finite or infinite path $e=\left(e_{i}\right)$ maximal (minimal) if every $e_{i}$ is maximal (minimal) amongst the edges from $r^{-1}\left(r\left(e_{i}\right)\right)$. A simple ordered Bratteli diagram $(B, \leq)$ is properly ordered if there are unique maximal and minimal infinite paths. Any simple stationary Bratteli diagram can be properly ordered. A Bratteli diagram $B=(V, E, \leq)$ is called stationary ordered if it is stationary and the partial linear order on $E_{n}$ does not depend on $n$. 
Let $(B, \leq)=(V, E, \leq)$ be a simple properly ordered stationary Bratteli diagram. Define a minimal homeomorphism $\phi_{B}: X_{B} \rightarrow X_{B}$ as follows. Let $\phi_{B}\left(x_{\max }\right)=$ $x_{\min }$. If $x=\left(x_{1}, x_{2}, \ldots\right) \neq x_{\max }$, let $k$ be the smallest number so that $x_{k}$ is not a maximal edge. Let $y_{k}$ be the successor of $x_{k}$ (hence $r\left(x_{k}\right)=r\left(y_{k}\right)$ ). Set $\phi_{B}(x)=\left(y_{1}, \ldots, y_{k-1}, y_{k}, x_{k+1}, x_{k+2}, \ldots\right)$, where $\left(y_{1}, \ldots, y_{k-1}\right)$ is the minimal path in $E\left(v_{0}, s\left(y_{k}\right)\right)$. The resulting minimal Cantor system $\left(X_{B}, \phi_{B}\right)$ is called a BratteliVershik system. If $\left(B^{\prime}, \leq^{\prime}\right)$ is a telescoping of $(B, \leq)$ which preserves the lexicographical order, then the Bratteli-Vershik systems $\left(X_{B}, \phi_{B}\right)$ and $\left(X_{B^{\prime}}, \phi_{B^{\prime}}\right)$ are isomorphic.

Definition 2.3. Let $B=(V, E)$ be a Bratteli diagram. Two infinite paths $x=\left(x_{i}\right)$ and $y=\left(y_{i}\right)$ from $X_{B}$ are called tail equivalent if there exists $i_{0}$ such that $x_{i}=y_{i}$ for all $i \geq i_{0}$. Denote by $\mathcal{R}$ the tail equivalence relation on $X_{B}$.

A Bratteli diagram is simple if the tail equivalence relation $\mathcal{R}$ is minimal; i.e. for arbitrary path $x \in X_{B}$ the set $\left\{y \in X_{B}: y\right.$ is tail equivalent to $\left.x\right\}$ is dense in $X_{B}$. Denote $X_{w}^{(n)}(\bar{e}):=\left\{x=\left(x_{i}\right) \in X_{B}: x_{i}=e_{i}, i=1, \ldots, n\right\}$, where $\bar{e}=\left(e_{1}, \ldots, e_{n}\right) \in$ $E\left(v_{0}, w\right), n \geq 1$. A measure $\mu$ on $X_{B}$ is called $\mathcal{R}$-invariant if for any two paths $\bar{e}$ and $\bar{e}^{\prime}$ from $E\left(v_{0}, w\right)$ and any vertex $w$, one has $\mu\left(X_{w}^{(n)}(\bar{e})\right)=\mu\left(X_{w}^{(n)}\left(\bar{e}^{\prime}\right)\right)$. The measure invariant for a stationary Bratteli-Vershik system is $\mathcal{R}$-invariant.

In the paper, we will consider only simple stationary Bratteli diagrams. Let $A=F^{T}$ be the matrix transpose to the incidence matrix of a diagram $B$. Let $\lambda$ be a Perron-Frobenius eigenvalue of $A$ and let $x=\left(x_{1}, \ldots, x_{K}\right)^{T}$ be the corresponding positive eigenvector such that $\sum_{i=1}^{K} x_{i}=1$. Suppose $B$ has no multiple edges between levels 0 and 1 . Then there is an ergodic probability measure $\mu$ defined by $\lambda$ and $x$ that satisfies the relation

$$
\mu\left(X_{i}^{(n)}(\bar{e})\right)=\frac{x_{i}}{\lambda^{n-1}},
$$

where $i \in V_{n}$ and $\bar{e}$ is a finite path with $r(\bar{e})=i$. Therefore, the clopen values set for $\mu$ has the form

$$
S(\mu)=\left\{\sum_{i=1}^{K} k_{i}^{(n)} \frac{x_{i}}{\lambda^{n-1}}: 0 \leq k_{i}^{(n)} \leq h_{i}^{(n)} ; n=1,2, \ldots\right\},
$$

where $h_{i}^{(n)}=\left|E\left(v_{0}, v_{i}\right)\right|, v_{i} \in V_{n}$. Let $H(x)$ be the additive subgroup of $\mathbb{R}$ generated by $x_{1}, \ldots, x_{K}$. We have $S(\mu)=\left(\bigcup_{N=0}^{\infty} \frac{1}{\lambda^{N}} H(x)\right) \cap[0,1]$ (see [2]). It is easy to see that $\lambda H(x) \subset H(x)$ and $\lambda^{m} \in H(x)$ for any $m \in \mathbb{N}$ (see [2]).

2.3. Substitution dynamical systems. Let $\mathcal{A}=\left\{a_{1}, \ldots, a_{s}\right\}$ be a finite alphabet. Let $\mathcal{A}^{*}$ be the collection of finite non-empty words over $\mathcal{A}$. Denote by $\Omega=\mathcal{A}^{\mathbb{Z}}$ the set of all two-sided infinite sequences on $\mathcal{A}$. A substitution $\sigma$ is a map $\sigma: \mathcal{A} \rightarrow \mathcal{A}^{*}$. It extends to maps $\sigma: \mathcal{A}^{*} \rightarrow \mathcal{A}^{*}$ and $\sigma: \Omega \rightarrow \Omega$ by concatenation. Denote by $T$ the shift on $\Omega: T\left(\ldots x_{-1} . x_{0} x_{1} \ldots\right)=\ldots x_{-1} x_{0} \cdot x_{1} \ldots$

Let $A_{\sigma}=\left(a_{i j}\right)_{i, j=1}^{s}$ be the incidence matrix associated to $\sigma$ where $a_{i j}$ is the number of occurrences of $a_{i}$ in $\sigma\left(a_{j}\right)$. Clearly, $A_{\sigma^{n}}=\left(A_{\sigma}\right)^{n}$ for every $n \geq 0$. A substitution $\sigma$ is called primitive if there is $n$ such that for each $a_{i}, a_{j} \in \mathcal{A}, a_{j}$ appears in $\sigma^{n}\left(a_{i}\right)$. Note that $\sigma$ is primitive if and only if $A_{\sigma}$ is a primitive matrix. If it happens that $|\sigma(a)|=q$ for any $a \in \mathcal{A}$, then the substitution $\sigma$ is said to be of constant length $q$. For $x \in \Omega$, let $L_{n}(x)$ be the set of all words of length $n$ occurring 
in $x$. Set $L(x)=\bigcup_{n \in \mathbb{N}} L_{n}(x)$. The language of $\sigma$ is the set $L_{\sigma}$ of all finite words occurring in $\sigma^{n}(a)$ for some $n \geq 0, a \in \mathcal{A}$. Set $X_{\sigma}=\left\{x \in \Omega: L(x) \subset L_{\sigma}\right\}$.

Throughout this paper we will consider only primitive substitutions $\sigma$ such that $X_{\sigma}$ is a Cantor set. The dynamical system $\left(X_{\sigma}, T_{\sigma}\right)$, where $T_{\sigma}$ is the restriction of $T$ to the $T$-invariant set $X_{\sigma}$, is called the substitution dynamical system associated to $\sigma$. It is well known (see [16]) that every primitive substitution generates a minimal and uniquely ergodic dynamical system.

The proofs of the following statements can be found in [16]. For every integer $p>0$ the substitution $\sigma^{p}$ defines the same language as $\sigma$, hence the systems $\left(X_{\sigma}, T_{\sigma}\right)$ and $\left(X_{\sigma^{p}}, T_{\sigma^{p}}\right)$ are isomorphic. Substituting $\sigma^{p}$ for $\sigma$ if needed, we can assume that there exist two letters $r, l \in \mathcal{A}$ such that $r$ is the last letter of $\sigma(r), l$ is the first letter of $\sigma(l)$ and $r l \in L_{\sigma}$. The sequence $\omega=\lim _{n \rightarrow \infty} \sigma^{n}(r . l) \in X_{\sigma}$ is a fixed point of $\sigma$ (that is, $\sigma(\omega)=\omega$ ) and $\omega_{-1}=r, \omega_{0}=l$. Then $X_{\sigma}=\overline{\operatorname{Orb}_{T}(\omega)}$.

The complexity of $u \in \Omega$ is the function $p_{u}(n)$ which associates to each integer $n \geq 1$ the cardinality of $L_{n}(u)$. It is easy to see that

$$
p_{u}(k+1)-p_{u}(k)=\sum_{w \in L_{k}(u)}\left(\operatorname{Card}\left\{a \in \mathcal{A}: w a \in L_{k+1}(u)\right\}-1\right) .
$$

The sequence $u$ is called minimal if every word occurring in $u$ occurs in an infinite number of places with bounded gaps. A fixed point of a primitive substitution is always minimal (see [8, 16]). Let $X_{u}$ be the set of all sequences $x \in \Omega$ such that $L_{n}(x)=L_{n}(u)$ for every $n \in \mathbb{N}$. For a primitive substitution $\sigma$ with the fixed point $u$, we have $X_{\sigma}=X_{u}=\overline{O r b_{T}(u)}$. Hence $p_{x}(n)=p_{u}(n)$ for every $n$ and every $x \in X_{u}$. Sometimes we will denote $p_{u}$ by $p_{\sigma}$ to stress that the complexity function is defined by $\sigma$.

The following results can be found in [6, 8, 16].

Theorem 2.4. (1) If the symbolic systems $\left(X_{u}, T\right)$ and $\left(X_{v}, T\right)$ associated to minimal sequences $u$ and $v$ are topologically conjugate, then there exists a constant $c$ such that, for all $n>c$,

$$
p_{u}(n-c) \leq p_{v}(n) \leq p_{u}(n+c) .
$$

Hence a relation $p_{u}(n) \leq a n^{k}+\bar{o}\left(n^{k}\right)$ when $n \rightarrow \infty$ is preserved by conjugacy (isomorphism).

(2) Let $\zeta$ be a primitive substitution and $p$ the complexity function of a fixed sequence $u=\zeta(u)$. Then, there exists a constant $C>0$ such that $p(n) \leq C n$ for every $n \geq 1$.

(3) Let $u \in\left\{a_{1}, \ldots, a_{s}\right\}^{\mathbb{Z}}$ and $p$ be the complexity function of $u$. Suppose that there exists $a>0$ such that $p(n) \leq$ an for all $n \geq n_{0}$. Then

$$
p(n+1)-p(n) \leq K s a^{3}
$$

for all $n \geq n_{0}$, where $K$ does not depend on $u$.

Definition 2.5. A substitution $\sigma$ on an alphabet $\mathcal{A}$ is called proper if there exists an integer $n>0$ and two letters $a, b \in \mathcal{A}$ such that for every $c \in \mathcal{A}, a$ is the first letter and $b$ is the last letter of $\sigma^{n}(c)$.

For every primitive substitution $\zeta$, there exists a proper substitution $\sigma$ such that the substitution systems $\left(X_{\zeta}, T_{\zeta}\right)$ and $\left(X_{\sigma}, T_{\sigma}\right)$ are isomorphic. The substitution $\sigma$ is built using the method of return words (see [7]). The following theorem establishes the link between incidence matrices of $\zeta$ and $\sigma$. 
Theorem 2.6 (17]). Let $\zeta$ be a non-proper primitive substitution and let $\lambda$ be the Perron-Frobenius eigenvalue of its incidence matrix $A_{\zeta}$. Let $\sigma$ be the corresponding proper substitution built by means of return words. Then the Perron-Frobenius eigenvalue of $A_{\sigma}$ is $\lambda^{k}$ for some $k \in \mathbb{N}$.

Stationary Bratteli diagrams are naturally related to substitution dynamical systems (primitive substitutions are considered in [7, [10, and the non-primitive case is studied in [3]). More precisely, let $(B, \leq)=(V, E, \leq)$ be a stationary ordered Bratteli diagram with no multiple edges between levels 0 and 1 . Choose a stationary labeling of $V_{n}$ by an alphabet $\mathcal{A}: V_{n}=\left\{v_{n}(a): a \in \mathcal{A}\right\}, n>0$. For $a \in \mathcal{A}$ consider the ordered set $\left(e_{1}, \ldots, e_{s}\right)$ of edges with range $v_{n}(a), n \geq 2$. Let $\left(a_{1}, \ldots, a_{s}\right)$ be the corresponding ordered set of the labels of the sources of these edges. The map $a \mapsto a_{1} \cdots a_{s}$ from $\mathcal{A}$ to $\mathcal{A}^{*}$ does not depend on $n$ and determines a substitution called the substitution read on $(B, \leq)$. Conversely, for any substitution dynamical system we can build the corresponding ordered stationary Bratteli diagram. The following theorem, proved in [7], shows the link between simple Bratteli diagrams and primitive substitution dynamical systems.

Theorem 2.7. Let $(B, \leq)$ be a stationary, properly ordered Bratteli diagram with only simple edges between the top vertex and the first level. Let $\sigma$ be the substitution read on $(B, \leq)$.

(i) If $\sigma$ is aperiodic, then the Bratteli-Vershik system $\left(X_{B}, \phi_{B}\right)$ is isomorphic to the substitution dynamical system $\left(X_{\sigma}, T_{\sigma}\right)$.

(ii) If $\sigma$ is periodic, the Bratteli-Vershik system $\left(X_{B}, \phi_{B}\right)$ is isomorphic to a stationary odometer.

In [17, the following result was proved:

Theorem 2.8. Let $\sigma$ be a primitive substitution whose incidence matrix has a natural Perron-Frobenius eigenvalue. Then $\left(X_{\sigma}, T_{\sigma}\right)$ is orbit equivalent to a stationary odometer system.

We will need the next result (see [7, 10]):

Theorem 2.9. Let $(B, \leq)$ be a stationary properly ordered Bratteli diagram. Then there exists a stationary properly ordered Bratteli diagram $\left(B^{\prime}, \leq^{\prime}\right)$ such that $B^{\prime}$ has no multiple edges between levels 0 and 1 and the systems $\left(X_{B}, \phi_{B}\right),\left(X_{B^{\prime}}, \phi_{B^{\prime}}\right)$ are isomorphic.

\section{ORBit EQUIVALENCE ClASS FOR A PRIMITIVE SUBSTITUTION}

Given a primitive proper substitution $\sigma$, we build countably many pairwise nonisomorphic substitution dynamical systems $\left\{\left(X_{\zeta_{n}}, T_{\zeta_{n}}\right)\right\}_{n=1}^{\infty}$ in the orbit equivalence class of $\left(X_{\sigma}, T_{\sigma}\right)$. Two essentially different constructions are elaborated. In the first one, we obtain countably many strong orbit equivalent substitution systems defined on the same alphabet. The second construction produces countably many orbit equivalent substitution systems with increasing cardinality of alphabets. Finally, given a primitive (not necessarily proper) substitution $\tau$, we find a stationary simple properly ordered Bratteli diagram with the least possible number of vertices such that the corresponding Bratteli-Vershik system is orbit equivalent to $\left(X_{\tau}, T_{\tau}\right)$.

Theorem 3.1. Let $\sigma$ be a primitive proper substitution. Let $(B, \leq)$ be the corresponding stationary properly ordered simple Bratteli diagram. Then there exist 
countably many telescopings $B_{n}$ of $B$ with proper orders $\leq_{n}$ and corresponding substitutions $\zeta_{n}$ read on $B_{n}$ such that the substitution dynamical systems $\left\{\left(X_{\zeta_{n}}, T_{\zeta_{n}}\right)\right\}_{n=1}^{\infty}$ are pairwise non-isomorphic and strong orbit equivalent to $\left(X_{\sigma}, T_{\sigma}\right)$.

Proof. Let $\mathcal{A}=\left\{a_{1}, \ldots, a_{s}\right\}$ be the alphabet for $\sigma$. Fix a number $l \in \mathbb{N}$. Let $\left\{\omega_{r}\right\}_{r=1}^{s^{l}}$ denote the set of all possible words of length $l$ over the alphabet $\mathcal{A}$. For any $N \in \mathbb{N}$ we can consider the telescoping $B_{N}$ of $B$ with respect to a sequence $\{k N\}_{k=0}^{\infty}$. Later in the proof we will choose some specific $N$. Let $F=A^{T}$ be the incidence matrix of $B$. Then $F^{N}$ is the incidence matrix of $B_{N}$. In our construction we will define a proper substitution $\zeta=\zeta(l)$ that is read on the Bratteli diagram $B_{N}$ whose incidence matrix is $A_{\zeta}=A^{N}$. This means that the number of occurrences of any letter $a_{i}$ in $\zeta\left(a_{j}\right)$ is known but we are free to choose any order of letters in the word $\zeta\left(a_{j}\right)$. In other words, we will change the lexicographical order $\leq$, which obviously determines $\sigma^{N}$, in order to define $\zeta$. Since the matrix $A$ is primitive, we can take $N=N(l)$ sufficiently large to guarantee that $\zeta$ satisfies the following conditions:

(1) for all $1 \leq j \leq s$ the word $\zeta\left(a_{j}\right)$ starts with the word $a_{1} a_{j}$ and ends with the letter $a_{1}$;

(2) the word $\zeta\left(a_{1}\right)$ contains as subwords all words $\left\{\omega_{i} a_{j}\right\}$ for $1 \leq i \leq s^{l}$ and $1 \leq j \leq s$.

Obviously, it follows that $\zeta$ is a proper substitution. The two substitution dynamical systems, $\left(X_{\zeta}, T_{\zeta}\right)$ and $\left(X_{\sigma}, T_{\sigma}\right)$, are strongly orbit equivalent. Indeed, if we consider the Bratteli diagrams corresponding to $\left(X_{\sigma^{N}}, T_{\sigma^{N}}\right)$ and $\left(X_{\zeta}, T_{\zeta}\right)$ as being unordered, they are identical. Hence the dimension groups associated to these minimal Cantor systems are order isomorphic by a map preserving the distinguished order unit (see Theorem 2.1 in [1] ).

We need to show that for an appropriate choice of $l$ the substitution $\zeta=\zeta(l)$ is such that the systems $\left(X_{\zeta(l)}, T_{\zeta(l)}\right)$ and $\left(X_{\sigma}, T_{\sigma}\right)$ are not isomorphic. We see that $\zeta^{\infty}\left(a_{1} \cdot a_{1}\right)=\lim _{n \rightarrow \infty} \zeta^{n}\left(a_{1} \cdot a_{1}\right)$ is a fixed point. The parameter $l$ should now be chosen in such a way that the complexity function $p_{\zeta}$ of $\zeta^{\infty}\left(a_{1} . a_{1}\right)$ grows essentially faster then the complexity function associated to $\left(X_{\sigma}, T_{\sigma}\right)$. We use the proof by contradiction. Suppose that for any $l$ the dynamical systems $\left(X_{\sigma}, T_{\sigma}\right)$ and $\left(X_{\zeta(l)}, T_{\zeta(l)}\right)$ are isomorphic. By Theorem 2.4 (2), there exists $C>0$ such that $p_{\sigma}(n) \leq C n$ for every $n \geq 1$. Then it follows from Theorem 2.4 (1) that $p_{\zeta}(n) \leq(C+1) n$ for sufficiently large $n$. Then, by Theorem 2.4 (3), we have $p_{\zeta}(n+1)-p_{\zeta}(n) \leq K s(C+1)^{3}$ for sufficiently large $n$, where $K$ is the universal constant which depends only on the slope $C+1$ (see [5]). We show that we can choose the parameter $l$ and an increasing sequence of natural numbers $\left\{n_{k}\right\}_{k=0}^{\infty}$ such that the difference $p_{\zeta}\left(n_{k}+1\right)-p_{\zeta}\left(n_{k}\right)$ is larger than $K s(C+1)^{3}$ for all $k \geq 0$. Thus, we get a contradiction.

To clarify the method of the proof, we first prove the theorem in the case when $\sigma$ is a substitution of constant length $q$. Then $\zeta$ is a substitution of length $q^{N}$.

By definition of $\zeta$, we have $p_{\zeta}(1)=s, p_{\zeta}(2)=s^{2}$ and $p_{\zeta}(k)=s^{k}$ for $1 \leq k \leq l+1$. The word $\zeta^{2}\left(a_{1}\right)$ contains the words $\zeta\left(\omega_{i}\right) \zeta\left(a_{j}\right)$ for $1 \leq i \leq s^{l}$ and $1 \leq j \leq s$. Recall that $\zeta\left(a_{j}\right)$ starts with $a_{1} a_{j}$. Thus, $\zeta^{2}\left(a_{1}\right)$ contains $s^{l}$ different words $\left\{\zeta\left(\omega_{i}\right) a_{1}\right\}_{i=1}^{s^{l}}$, and each word can be followed by any letter from $\mathcal{A}$. Since $l q+1=\left|\zeta\left(\omega_{i}\right)\right|+\left|a_{1}\right|$, we obtain $p_{\zeta}(l q+2)-p_{\zeta}(l q+1) \geq s^{l}(s-1)$ by (2.1). 
Consider $\zeta^{3}\left(a_{1}\right)$. Then apply the previous arguments with $\zeta\left(a_{i}\right)$ instead of $a_{i}$. Since $\left|\zeta^{2}\left(\omega_{i}\right)\right|+\left|\zeta\left(a_{1}\right)\right|+\left|a_{1}\right|=l q^{2}+q+1$, we get $p_{\zeta}\left(l q^{2}+q+2\right)-p_{\zeta}\left(l q^{2}+q+1\right) \geq$ $s^{l}(s-1)$. Thus, we conclude by induction that for all $m \in \mathbb{N}$,

$$
p_{\zeta}\left(l q^{m}+\sum_{i=0}^{m-1} q^{i}+1\right)-p_{\zeta}\left(l q^{m}+\sum_{i=0}^{m-1} q^{i}\right) \geq s^{l}(s-1) .
$$

Taking $l$ large enough, we can make the difference $p_{\zeta}(k+1)-p_{\zeta}(k)$ arbitrarily large for an infinite number of values of $k$.

To prove the theorem in the general case, denote $q_{m}=\left|\zeta^{m}\left(a_{1}\right)\right|$ for $m \in \mathbb{N}$. Let $D_{m}=\max _{1 \leq i \leq s}\left|\zeta^{m}\left(a_{i}\right)\right|$ and $d_{m}=\min _{1 \leq i \leq s}\left|\zeta^{m}\left(a_{i}\right)\right|$. Since $\left|\omega_{i}\right|=l$, we have $l d_{m} \leq\left|\zeta^{m}\left(\omega_{i}\right)\right| \leq l D_{m}$ for any $1 \leq i \leq s^{l}$ and $m \geq 1$. The matrix $A_{\zeta}$ is strictly positive; hence there exist positive constants $M_{1}, M_{2}$ such that for every $m \geq 1$ we have $M_{1} \lambda^{m} \leq d_{m} \leq D_{m} \leq M_{2} \lambda^{m}$, where $\lambda$ is the Perron-Frobenius eigenvalue of $A_{\zeta}$ (see [16]). Hence $\frac{D_{m}}{d_{m}} \leq \frac{M_{2}}{M_{1}}$ for all $m \geq 1$. For $r>0$, set $l=l(r)=\left(\left[\frac{M_{2}}{M_{1}}\right]+1\right) r$. Then $l \geq r \frac{M_{2}}{M_{1}} \geq r \frac{D_{m}}{d_{m}}$ and $l d_{m} \geq r D_{m}$ for all $m \geq 1$. Thus, $\left\{\zeta^{m}\left(\omega_{i}\right)\right\}_{i=1}^{s^{l}}$ contains at least $s^{r}$ different suffixes of length $D_{m} r$ and each suffix can be followed by any word from $\left\{\zeta^{m}\left(a_{j}\right)\right\}_{j=1}^{s}$. By the same argument as in the case of substitution of constant length, we conclude that

$$
p_{\zeta}\left(D_{m} r+\sum_{i=0}^{m-1} q_{i}+1\right)-p_{\zeta}\left(D_{m} r+\sum_{i=0}^{m-1} q_{i}\right) \geq s^{r}(s-1) .
$$

Thus, the systems $\left(X_{\sigma}, T_{\sigma}\right)$ and $\left(X_{\zeta}, T_{\zeta}\right)$ are not isomorphic.

Recall that $p_{\sigma}(n) \leq C n$ for all $n \geq 1$ and the parameter $l$ is such that the inequality $p_{\zeta(l)}(n) \leq(C+1) n$ does not hold for infinitely many values of $n$. Therefore, for any $N \in \mathbb{N}$ there exists $M>N$ such that $p_{\zeta}(M)>(C+1) M$. Set $\zeta_{1}=\zeta$. There exists $C_{1}>0$ such that $p_{\zeta_{1}}(n) \leq C_{1} n$ for every $n \geq 1$. By the same method as above, we construct $\zeta_{m+1}$ using $\zeta_{m}$ for $m \in \mathbb{N}$. We obtain $p_{\zeta_{m}}(n) \leq C_{m} n$ for $n \geq 1$, and for any $N \in \mathbb{N}$ there exists $M>N$ such that $p_{\zeta_{m+1}}(M)>\left(C_{m}+1\right) M$. Hence, by Theorem 2.4. we obtain countably many pairwise non-isomorphic substitution dynamical systems $\left\{\left(X_{\zeta_{m}}, T_{\zeta_{m}}\right)\right\}_{m=1}^{\infty}$ in the strong orbit equivalence class of $\left(X_{\sigma}, T_{\sigma}\right)$.

In contrast to the first construction, where the cardinality of the alphabet was fixed and which led us to a class of strongly orbit equivalent substitution systems, we will now consider a class of substitution dynamical systems defined on the alphabets of variable cardinality.

Recall that for a vector $x$, we denote by $H(x)$ the additive subgroup generated by the coordinates of $x$. For a primitive matrix $A \in \operatorname{Mat}(\mathbb{N}, s)$ with the PerronFrobenius eigenvalue $\lambda$ and the corresponding normalized eigenvector $x$, we denote it by $S(A)=\bigcup_{N=0}^{\infty} \frac{1}{\lambda^{N}} H(x)$.

Lemma 3.2. Let $A \in \operatorname{Mat}(\mathbb{N}, s)$ be a primitive matrix. Then there exist primitive matrices $\left\{A_{n}\right\}_{n=1}^{\infty}$, where $A_{n} \in \operatorname{Mat}(\mathbb{N}, s+n)$ such that $S(A)=S\left(A_{n}\right)$ for all $n \in \mathbb{N}$.

Proof. Let $\lambda$ be the Perron-Frobenius eigenvalue of $A$ and $x=\left(x_{1}, \ldots, x_{s}\right)^{T}$ the corresponding normalized eigenvector. We construct an $(s+1) \times(s+1)$ matrix $A_{1}$ such that $A_{1}$ satisfies the condition of the lemma and $y=\left(x_{1}, \ldots, x_{s}, \lambda-1\right)^{T}$ 
is the Perron-Frobenius eigenvector of $A_{1}$. Since $x$ is the normalized eigenvector of $A$, we have $\sum_{j=1}^{s} a_{i j} x_{j}=\lambda x_{i}$ and $\sum_{i=1}^{s} \sum_{j=1}^{s} a_{i j} x_{j}=\lambda$. Then

$$
\lambda-1=\sum_{j=1}^{s} x_{j}\left(\sum_{i=1}^{s} a_{i j}-1\right) \in H\left(x_{1}, \ldots, x_{s}\right) .
$$

Denote by $a_{i j}^{(k)}$ the entries of the matrix $A^{k}$. We have

$$
\lambda^{k} x_{i}=\sum_{j=1}^{s} a_{i j}^{(k)} x_{j}=\sum_{j=1}^{s}\left(a_{i j}^{(k)}-\left(\sum_{l=1}^{s} a_{l j}-1\right)\right) x_{j}+\lambda-1 .
$$

Clearly, all the coefficients $a_{i j}^{(k)}-\left(\sum_{l=1}^{s} a_{l j}-1\right)$ are positive integers for sufficiently large $k$. We have $\lambda^{k}(\lambda-1)=\sum_{j=1}^{s}\left(\sum_{i=1}^{s}\left(a_{i j}^{(k+1)}-a_{i j}^{(k)}\right)\right) x_{j}$. It is obvious that the numbers $\sum_{i=1}^{s}\left(a_{i j}^{(k+1)}-a_{i j}^{(k)}\right)$ are positive integers for all $k \in \mathbb{N}$ and $1 \leq j \leq s$. Hence we define

$$
A_{1}=\left(\begin{array}{cccc}
a_{11}^{(k)}-\left(\sum_{i=1}^{s} a_{i 1}-1\right) & \ldots & a_{1 n}^{(k)}-\left(\sum_{i=1}^{s} a_{i, n}-1\right) & 1 \\
\vdots & \ddots & \vdots & \vdots \\
a_{n 1}^{(k)}-\left(\sum_{i=1}^{s} a_{i 1}-1\right) & \ldots & a_{n n}^{(k)}-\left(\sum_{i=1}^{s} a_{i, n}-1\right) & 1 \\
\sum_{i=1}^{s}\left(a_{i 1}^{(k+1)}-a_{i 1}^{(k)}\right) & \ldots & \sum_{i=1}^{s}\left(a_{i, n}^{(k+1)}-a_{i, n}^{(k)}\right) & 0
\end{array}\right) .
$$

It is straightforward to check that $A_{1} y=\lambda^{k} y$. Since $\lambda-1 \in H\left(x_{1}, \ldots, x_{s}\right)$, we have $H\left(y_{1}, \ldots, y_{s+1}\right)=H\left(x_{1}, \ldots, x_{s}\right)$. The normalized Perron-Frobenius eigenvector of $A_{1}$ is $z=\frac{1}{\lambda} y$. Since $\lambda H(x) \subset H(x)$, we see that $S(A)=S\left(A_{1}\right)$.

To complete the proof, we note that the construction of $A_{n+1}$ uses $A_{n}$ in the same way as the construction of $A_{1}$ uses $A$.

Theorem 3.3. Let $\sigma$ be a proper substitution. Then there exist countably many proper substitutions $\left\{\zeta_{n}\right\}_{n=1}^{\infty}$ such that $\left(X_{\sigma}, T_{\sigma}\right)$ is orbit equivalent to $\left(X_{\zeta_{n}}, T_{\zeta_{n}}\right)$ but the systems $\left\{\left(X_{\zeta_{n}}, T_{\zeta_{n}}\right)\right\}_{n=1}^{\infty}$ are pairwise non-isomorphic.

Proof. Let $A$ be the incidence matrix of substitution $\sigma$ defined on an alphabet $\left\{a_{1}, \ldots, a_{s}\right\}$. Let $\lambda$ be the Perron-Frobenius eigenvalue of $A$ and $x=\left(x_{1}, \ldots, x_{s}\right)^{T}$ be the normalized Perron-Frobenius eigenvector. By Theorem 2.4, there exists $C>0$ such that $p_{\sigma}(n) \leq C n$ for every $n \geq 1$. We can assume $C \in \mathbb{N}$ and $C+1>s$. By Lemma 3.2 there exists a primitive matrix $\widetilde{A} \in \operatorname{Mat}(\mathbb{N}, C+2)$ such that $S(\widetilde{A})=S(A)$. We define a primitive substitution $\zeta$ on the alphabet $\left\{a_{1}, \ldots, a_{C+2}\right\}$ such that $A_{\zeta}=\widetilde{A}$. We can always assume that $(\widetilde{A})_{1 i} \geq 2$ for $i=1, \ldots, C+2$; otherwise we would take the power of $\widetilde{A}$ instead of $\widetilde{A}$. We require also that the word $\zeta\left(a_{j}\right)$ starts with the letters $a_{1} a_{j}$ and ends with the letter $a_{1}$ for all $j=1, \ldots, C+2$. Then $\zeta$ is a proper primitive substitution.

Denote by $u=\lim _{n \rightarrow \infty} \zeta^{n}\left(a_{1} . a_{1}\right)$ the unique fixed point for $\zeta$. Since $\zeta$ is primitive, the sequence $u$ contains $\zeta^{k}\left(a_{j}\right)=\zeta^{k-1}\left(a_{1}\right) \zeta^{k-2}\left(a_{1}\right) \ldots \zeta\left(a_{1}\right) a_{1} a_{j} \ldots a_{1}$ as a subword for $k \geq 1$ and $j=1, \ldots, C+2$. We have $p_{u}(1)=C+2$. Since all letters $\left\{a_{j}\right\}_{j=1}^{C+2}$ can follow the word $\zeta^{k-1}\left(a_{1}\right) \zeta^{k-2}\left(a_{1}\right) \ldots \zeta\left(a_{1}\right) a_{1}$ for any $k$, we have 
$p_{u}(n+1)-p_{u}(n) \geq C+1$ for $n \geq 1$ by (2.1). It follows that $p_{u}(n)>(C+1) n$ for all $n \geq 1$. Hence, by Theorem 2.4 , the systems $\left(X_{\sigma}, T_{\sigma}\right)$ and $\left(X_{\zeta}, T_{\zeta}\right)$ are not isomorphic.

We will apply induction to produce a needed sequence of substitutions. For $\zeta_{1}:=$ $\zeta$, there exists $C_{1}>0$ such that $p_{\zeta_{1}}(n) \leq C_{1} n$ for every $n \geq 1$. By the same method as above, we can construct $\zeta_{m+1}$ using $\zeta_{m}$ for $m \in \mathbb{N}$ such that $S\left(A_{\zeta_{m}}\right)=S(A)$. We obtain $C_{m} n \leq p_{\zeta_{m+1}}(n) \leq C_{m+1} n$ for $m \in \mathbb{N}$ and $n \geq 1$. Hence, by Theorem 2.4 and Lemma 3.2, we obtain countably many pairwise non-isomorphic substitution dynamical systems $\left\{\left(X_{\zeta_{m}}, T_{\zeta_{m}}\right)\right\}_{m=1}^{\infty}$ in the orbit equivalence class of $\left(X_{\sigma}, T_{\sigma}\right)$.

In the case of a primitive substitution, we find a stationary simple properly ordered Bratteli diagram with the least possible number of vertices such that the corresponding dynamical systems are orbit equivalent. In the case of a primitive substitution whose incidence matrix has a natural Perron-Frobenius eigenvalue, the corresponding diagram has only one vertex on each level (see Theorem 2.8). We recall some notions and results from $[2$.

Let $A \in \operatorname{Mat}(\mathbb{N}, s)$ be a primitive matrix. Let $\lambda$ be a Perron-Frobenius eigenvalue of $A$ and $x=\left(x_{1}, \ldots, x_{s}\right)^{T}$ be the normalized Perron-Frobenius eigenvector. Denote by $k$ the degree of the algebraic integer $\lambda$. The number field $\mathbb{Q}(\lambda)$ is a subfield of $\mathbb{R}$ whose elements are written as $\left\{a_{0}+a_{1} \lambda+\ldots+a_{k-1} \lambda^{k-1}: a_{0}, a_{1}, \ldots, a_{k-1} \in \mathbb{Q}\right\}$. The numbers $1, \lambda, \ldots, \lambda^{k-1}$ form a basis of $\mathbb{Q}(\lambda)$ as a vector space over $\mathbb{Q}$. If we need to emphasize that a real number $y=a_{0}+a_{1} \lambda+\ldots+a_{k-1} \lambda^{k-1} \in \mathbb{Q}(\lambda)$ is considered as a vector $\left(a_{0}, a_{1}, \ldots, a_{k-1}\right)^{T} \in \mathbb{Q}^{k}$, we will use the notation y. Let $\mathbf{n}$ denote the vector $\left(1, \lambda, \ldots, \lambda^{k-1}\right)^{T} \in \mathbb{R}^{k}$. Then, for any $\mathbf{y} \in \mathbb{Q}^{k}$, the corresponding number $y \in \mathbb{R}$ can be written as $y=\langle\mathbf{y}, \mathbf{n}\rangle$.

Let $B$ be a stationary simple Bratteli diagram with incidence matrix $F=A^{T}$ and no multiple edges between levels 0 and 1 . Let $\mu$ be its unique ergodic probability $\mathcal{R}$-invariant measure. Let $G(S(\mu))$ be an additive subgroup of reals generated by $S(\mu)$. It is not hard to see that $S(A)=G(S(\mu)) \subset \mathbb{Q}(\lambda)$.

Let $p(\lambda)$ be the polynomial in $\mathbb{Q}(\lambda)$ such that $\lambda^{-1}=p(\lambda)$. The map $y \mapsto p(\lambda) y$ in $\mathbb{Q}(\lambda)$ determines a linear transformation in the vector space $\mathbb{Q}^{k}$. Let $D$ be the matrix which corresponds to this transformation. The following results can be found in the proof of Theorem 3.2 in $[2$. The matrix $D \in \operatorname{Mat}(\mathbb{Q}, k)$ is a non-singular matrix, and for any $\mathbf{x} \in \mathbb{Q}^{k}$ the vector $D \mathbf{x} \in \mathbb{Q}^{k}$ corresponds to the number $\frac{x}{\lambda} \in \mathbb{R}$. Let $C=D^{-1}$. The matrix $C$ has only one eigenvector $\mathbf{y}_{1}$ with eigenvalue $\lambda$, the absolute value of any other eigenvalue of $C$ is less than $\lambda$, and $\mathbf{y}_{1}$ is the only eigenvector of $C$ that is not orthogonal to $\mathbf{n}$. Obviously, we can assume $\left\langle\mathbf{y}_{1}, \mathbf{n}\right\rangle>0$. Denote by $\pi=\left\{\mathbf{y} \in \mathbb{R}^{k}:\langle\mathbf{y}, \mathbf{n}\rangle=0\right\}$. The iterations of $C$ drive any ray which is not in $\pi$ to the limit ray generated by $\mathbf{y}_{1}$; the iterations of $D=C^{-1}$ do the opposite. More precisely, if $\langle\mathbf{y}, \mathbf{n}\rangle \neq 0$ for some $\mathbf{y} \in \mathbb{Q}^{k}$, then the angle between the line generated by $D^{N} \mathbf{y}$ and $\pi$ can be made arbitrarily small when $N$ tends to infinity.

Remark 3.4. For $A \in \operatorname{Mat}(\mathbb{N}, s)$, let $\lambda$ be the Perron-Frobenius eigenvalue for $A$ and $x=\left(x_{1}, \ldots, x_{s}\right)^{T}$ be the corresponding eigenvector. Then $\operatorname{deg} \lambda=\operatorname{deg} \lambda^{m}$ for all $m \in \mathbb{N}$. Indeed, since the Perron-Frobenius eigenvalue of $A^{m}$ is $\lambda^{m}$ and $A^{m} x=\lambda^{m} x$, we have $\operatorname{deg} \lambda^{m}=\operatorname{dim}\left(\operatorname{Lin}_{\mathbb{Q}}\left\{\mathbf{x}_{1}, \ldots, \mathbf{x}_{k}\right\}\right)=\operatorname{deg} \lambda$. 
Lemma 3.5. Let $A \in \operatorname{Mat}(\mathbb{N}, s)$ be a primitive matrix. Let $\lambda$ be the PerronFrobenius eigenvalue of $A$. Let $k \geq 2$ be the degree of algebraic number $\lambda$. Then there exists a primitive matrix $\widetilde{A} \in \operatorname{Mat}(\mathbb{N}, k)$ such that $S(\widetilde{A})=\alpha S(A)$ for some positive $\alpha \in \mathbb{Q}(\lambda)$. Moreover, $k$ is the least possible dimension for which this equality holds.

Proof. Let $x=\left(x_{1}, \ldots, x_{s}\right)^{T}$ be the normalized Perron-Frobenius eigenvector of $A$. Let $\Lambda\left(\mathbf{x}_{1}, \ldots, \mathbf{x}_{s}\right)$ be the lattice in $\mathbb{R}^{k}$ generated by $\mathbf{x}_{1}, \ldots, \mathbf{x}_{s}$. Then $\Lambda\left(\mathbf{x}_{1}, \ldots, \mathbf{x}_{s}\right) \subset$ $\mathbb{Q}^{k}$ corresponds to the group $H\left(x_{1}, \ldots, x_{s}\right) \subset \mathbb{R}$. There exist $\mathbf{f}_{1}, \ldots, \mathbf{f}_{k} \in \mathbb{Q}^{k}$ such that $\Lambda\left(\mathbf{f}_{1}, \ldots, \mathbf{f}_{k}\right)=\Lambda\left(\mathbf{x}_{1}, \ldots, \mathbf{x}_{s}\right)$ (see 15]). Since $1, \lambda, \ldots, \lambda^{k-1} \in H(x)$, the vectors $\left\{\mathbf{f}_{i}\right\}_{i=1}^{k}$ form a basis of $\mathbb{Q}^{k}$. By changing $\mathbf{f}_{i}$ to $-\mathbf{f}_{i}$, we can make all vectors $\left\{\mathbf{f}_{i}\right\}_{i=1}^{k}$ satisfy the inequality $\left\langle\mathbf{f}_{i}, \mathbf{n}\right\rangle>0$. Since $D$ is a non-singular linear transformation of $\mathbb{Q}^{k}$, the vectors $\left\{D^{N} \mathbf{f}_{i}\right\}_{i=1}^{k}$ form a basis of $\mathbb{Q}^{k}$ for every $N \in \mathbb{N}$ and $\Lambda\left(D^{N} \mathbf{f}_{1}, \ldots, D^{N} \mathbf{f}_{k}\right)=\Lambda\left(D^{N} \mathbf{x}_{1}, \ldots, D^{N} \mathbf{x}_{s}\right)$. Consider the cone

$$
K\left(D^{N} \mathbf{f}_{1}, \ldots, D^{N} \mathbf{f}_{k}\right)=\left\{\sum_{i=1}^{k} \beta_{i} D^{N} \mathbf{f}_{i}: \beta_{i} \geq 0, i=1, \ldots, k\right\} .
$$

Recall that $\mathbf{y}_{1}$ is an eigenvector of $C$ such that $\left\langle\mathbf{y}_{1}, \mathbf{n}\right\rangle>0$ and the iterations of $C$ drive any ray which is not in $\pi$ to the limit ray generated by $\mathbf{y}_{1}$. There exists $N \in \mathbb{N}$ such that the vectors $\mathbf{y}_{1}, \mathbf{x}_{1}, \ldots, \mathbf{x}_{s}$ lie in the cone $K\left(D^{N} \mathbf{f}_{1}, \ldots, D^{N} \mathbf{f}_{k}\right)$. Then there exists an integer $M>0$ such that $D^{N-M} \mathbf{f}_{i} \in K\left(D^{N} \mathbf{f}_{1}, \ldots, D^{N} \mathbf{f}_{k}\right)$ for $i=1, \ldots, k$. Since $\lambda H(x) \subset H(x)$, we also have $D^{N-M} \mathbf{f}_{i} \in \Lambda\left(D^{N} \mathbf{f}_{1}, \ldots, D^{N} \mathbf{f}_{k}\right)$ for $i=1, \ldots, k$. Since $\mathbf{f}_{1}, \ldots, \mathbf{f}_{k}$ are linearly independent, there exist positive integers $\left\{\tilde{a}_{i j}\right\}_{i, j=1}^{k}$ such that $D^{N-M} \mathbf{f}_{i}=\sum_{j=1}^{k} \tilde{a}_{i j} D^{N} \mathbf{f}_{j}$ for $i=1, \ldots, k$. Set $z_{i}=\left\langle D^{N} \mathbf{f}_{i}, \mathbf{n}\right\rangle$ and $\widetilde{A}=\left(\tilde{a}_{i j}\right)_{i, j=1}^{k}$. Then

$$
z=\left(\frac{z_{1}}{\sum_{l=1}^{k} z_{k}}, \ldots, \frac{z_{k}}{\sum_{l=1}^{k} z_{k}}\right)^{T} \in \mathbb{R}^{k}
$$

is a normalized Perron-Frobenius eigenvector for $\widetilde{A}$ with eigenvalue $\lambda^{M}$. Setting $\alpha=$ $\sum_{l=1}^{k} z_{k}$, we obtain that $\alpha S(A)=S(\widetilde{A})$ because $H\left(z_{1}, \ldots, z_{k}\right)=\frac{1}{\lambda^{N}} H\left(x_{1}, \ldots, x_{s}\right)$.

Now we show that if $P \in \operatorname{Mat}(\mathbb{N}, l)$ such that $S(P)=\alpha S(A)$ for some $\alpha \in \mathbb{R}$, then $l \geq k$. First, we show that $\operatorname{deg} \beta=d \geq k$. Assume that the converse holds. Suppose $\operatorname{deg} \beta=d<k$. Recall that $1, \lambda, \ldots, \lambda^{k-1} \in H\left(x_{1}, \ldots, x_{s}\right)$. We have $S(P) \subset \mathbb{Q}(\beta)$. Then the elements of $S(P)=\alpha S(A)$ can be represented as some vectors of $\mathbb{Q}^{d}$. In particular, $\alpha, \alpha \lambda, \ldots, \alpha \lambda^{k-1}$ can be represented as $\mathbf{v}_{1}, \ldots, \mathbf{v}_{k} \in$ $\mathbb{Q}^{d}$. Since $d<k$, the vectors $\left\{\mathbf{v}_{i}\right\}_{i=1}^{k}$ are linearly dependent over $\mathbb{Q}$; hence there exist rational numbers $\left\{r_{i}\right\}_{i=1}^{k}$ such that $\sum_{i=1}^{k} r_{i} \mathbf{v}_{i}=0$. Returning from $\mathbb{Q}^{d}$ to $\mathbb{R}$ we obtain $\alpha \sum_{i=1}^{k} r_{i} \lambda^{i}=0$. But then the algebraic degree of $\lambda$ is less than $k$. This is a contradiction, and hence $d \geq k$. Since $\beta$ is a root of characteristic polynomial for $P$, the dimension of $P$ is not less than $k$.

Remark 3.6. Given a Perron number $\lambda$ with $\operatorname{deg} \lambda=k$, we show that there exists a primitive matrix $\widetilde{A} \in \operatorname{Mat}(\mathbb{N}, k)$ such that $\lambda^{M}$ is the Perron-Frobenius eigenvalue of $\widetilde{A}$ for some $M \in \mathbb{N}$. In 13 , it was shown that there may not exist a matrix $\widetilde{A} \in \operatorname{Mat}(\mathbb{N}, k)$ with the Perron-Frobenius eigenvalue $\lambda$ (see Example 3.13 below).

Recall that a stationary Bratteli diagram may have multiple edges between levels 0 and 1 . The following theorem is a generalization of Theorem 2.8 
Theorem 3.7. Let $\sigma$ be a primitive substitution whose incidence matrix has a Perron-Frobenius eigenvalue $\lambda$ and $k=\operatorname{deg} \lambda$. Then $\left(X_{\sigma}, T_{\sigma}\right)$ is orbit equivalent to a Bratteli-Vershik system defined on a stationary Bratteli diagram with $k$ vertices on each level. Moreover, there is no stationary Bratteli-Vershik system with less than $k$ vertices which is orbit equivalent to $\left(X_{\sigma}, T_{\sigma}\right)$.

Proof. Suppose $\sigma$ is not a proper substitution. Then, by Theorem 2.6. there exists a proper substitution $\zeta$ with incidence matrix $A_{\zeta}$ such that $\left(X_{\zeta}, T_{\zeta}\right)$ is isomorphic to $\left(X_{\sigma}, T_{\sigma}\right)$ and the Perron-Frobenius eigenvalue of $A_{\zeta}$ is $\lambda^{d}$ for some $d \in \mathbb{N}$. By Remark 3.4, we have $\operatorname{deg} \lambda=\operatorname{deg} \lambda^{d}=k$. Thus, without loss of generality, we may assume that $\sigma$ is a proper substitution.

We will use the notation from Lemma 3.5. Let $A$ be the incidence matrix for $\sigma$. There exist a primitive matrix $\widetilde{A} \in \operatorname{Mat}(\mathbb{N}, k)$ and a positive number $\alpha \in \mathbb{Q}(\lambda)$ such that $S(\widetilde{A})=\alpha S(A)$. Let $(B, \leq)$ be a stationary ordered Bratteli diagram corresponding to $\sigma$ and let $\mu$ be the unique invariant measure for the Bratteli-Vershik system $\left(X_{B}, \phi_{B}\right)$. Suppose $\widetilde{B}$ is the stationary Bratteli diagram with incidence matrix $\widetilde{F}=\widetilde{A}^{T}$ and no multiple edges between levels 0 and 1 . Let $x=\left(x_{1}, \ldots, x_{s}\right)^{T}$ be a normalized Perron-Frobenius eigenvector for $A$. The diagram $\widetilde{B}$ has $k$ vertices and $\frac{1}{\alpha}\left(\left\langle D^{N} \mathbf{f}_{1}, \mathbf{n}\right\rangle, \ldots,\left\langle D^{N} \mathbf{f}_{k}, \mathbf{n}\right\rangle\right)^{T}$ is the normalized Perron-Frobenius eigenvector for $\widetilde{A}$. Since the vectors $\mathbf{x}_{1}, \ldots, \mathbf{x}_{s}$ lie in the positive cone $K\left(D^{N} \mathbf{f}_{1}, \ldots, D^{N} \mathbf{f}_{k}\right)$ and in the lattice $\Lambda\left(D^{N} \mathbf{f}_{1}, \ldots, D^{N} \mathbf{f}_{k}\right)$, each $\mathbf{x}_{i}$ is a linear combination of $\left\{D^{N} \mathbf{f}_{j}\right\}_{j=1}^{k}$ with natural coefficients. We make a finite change between the zero and first levels of $\widetilde{B}$ and obtain $B_{1}$ as follows. If $\mathbf{x}_{i}=\sum_{j=1}^{k} b_{i j} D^{N} \mathbf{f}_{j}$, then let $B_{1}$ have $\sum_{i=1}^{s} b_{i j}$ edges between $v_{0}$ and the $j$-th vertex of the first level. Let $\leq_{1}$ be a proper order on $B_{1}$. Let $\nu$ be the unique invariant measure for $\left(X_{B_{1}}, \phi_{B_{1}}\right)$. Then $\left(B_{1}, \leq_{1}\right)$ is a stationary Bratteli diagram such that $G(S(\nu))=G(S(\mu))$. Hence the BratteliVershik system on $\left(B_{1}, \leq_{1}\right)$ is orbit equivalent to $\left(X_{\sigma}, T_{\sigma}\right)$. Let $\widetilde{\leq}$ be any proper order on $\widetilde{B}$. Note that the proper substitution systems associated to $(B, \leq)$ and $(\widetilde{B}, \widetilde{\leq})$ are Kakutani orbit equivalent. Indeed, two Bratteli-Vershik systems associated to properly ordered Bratteli diagrams are Kakutani equivalent if and only if one diagram can be obtained from the other by a finite change, i.e. by doing a finite number of finite telescopings and adding and/or removing a finite number of edges (see [7]). By Lemma 3.5 there is no stationary Bratteli-Vershik system on the diagram with less than $k$ vertices which is orbit equivalent to $\left(X_{\sigma}, T_{\sigma}\right)$.

Remark 3.8. The diagram $\left(B_{1}, \leq_{1}\right)$ has multiple edges between levels 0 and 1 . By Theorem 2.9, there exists a stationary Bratteli diagram $\left(B_{2}, \leq_{2}\right)$ such that $B_{2}$ has no multiple edges between levels 0 and 1 , and Bratteli-Vershik systems $\left(X_{B_{1}}, \phi_{B_{1}}\right)$, $\left(X_{B_{2}}, \phi_{B_{2}}\right)$ are isomorphic. Let $A_{2}$ be the matrix transpose to the incidence matrix of $B_{2}$. Then $S\left(A_{2}\right)=S(A)$.

The corollary easily follows from the proof of Theorem 3.7 and Remark 3.8

A Perron number is a real algebraic integer greater than one, that is, larger than the absolute value of any of its Galois conjugates [13].

Corollary 3.9. Let $\lambda \in \mathbb{R}$ be a Perron number and $\left\{x_{i}\right\}_{i=1}^{s} \subset \mathbb{Q}(\lambda) \cap(0, \infty)$ with $\sum_{i=1}^{s} x_{i}=1$. Let $H=H\left(x_{1}, \ldots, x_{s}\right)$ be the additive group generated by $x_{1}, \ldots, x_{s}$. Suppose $\left\{\boldsymbol{x}_{i}\right\}_{i=1}^{s}$ are vectors in $\mathbb{Q}^{k}$ corresponding to $\left\{x_{i}\right\}_{i=1}^{s}$ and $\operatorname{Lin}_{\mathbb{Q}}\left\{\boldsymbol{x}_{i}\right\}_{i=1}^{s}$ denotes the set of all rational linear combinations of $\left\{\boldsymbol{x}_{i}\right\}_{i=1}^{s}$. 
(i) If $\operatorname{Lin}_{\mathbb{Q}}\left\{\boldsymbol{x}_{i}\right\}_{i=1}^{s}=\mathbb{Q}^{k}$ and $\lambda^{M} H \subset H$ for some $M \in \mathbb{N}$, then there exists a primitive matrix $A$ with natural entries such that $S(A)=\bigcup_{N=0}^{\infty} \frac{1}{\lambda^{N}} H\left(x_{1}, \ldots, x_{s}\right)$.

(ii) If $\lambda H \subset H$, then there exists a primitive matrix $A$ with natural entries such that $S(A)=\bigcup_{N=0}^{\infty} \frac{1}{\lambda^{N}} H\left(x_{1}, \ldots, x_{s}\right)$.

Proposition 3.10. Let $\lambda \in \mathbb{Q}$ and $\left\{x_{i}\right\}_{i=1}^{s} \subset \mathbb{Q} \cap(0, \infty)$ with $\sum_{i=1}^{s} x_{i}=1$. Let $H(x)=H\left(x_{1}, \ldots, x_{s}\right)$ be an additive group generated by $x_{1}, \ldots, x_{s}$. Let $Y=$ $\bigcup_{l \in \mathbb{N}}\left\{\left\{y_{j}\right\}_{j=1}^{l} \subset \mathbb{Q} \cap(0, \infty): \sum_{j=1}^{l} y_{j}=1\right.$ and $\left.H(y)=H(x)\right\}$. Then $Y$ is a finite set.

Proof. Let $x_{i}=\frac{p_{i}}{q}$, where $\operatorname{gcd}\left(p_{1}, \ldots, p_{s}\right)=1$. We have $H(x)=\frac{1}{q} \mathbb{Z}$. Then $Y=\bigcup_{l \in \mathbb{N}}\left\{\left\{y_{j}\right\}_{j=1}^{l} \subset \mathbb{Q} \cap[0, \infty): \sum_{j=1}^{l} y_{j}=1\right.$ and $\left.H(y)=\frac{1}{q} \mathbb{Z}\right\}$. Hence $y_{i}=\frac{q_{i}}{q}$ for some $q_{i} \in \mathbb{N}$ such that $\sum_{i=1}^{l} q_{i}=q$. Since the number of partitions of $q$ into natural numbers is finite, we obtain $|Y|<\infty$.

More results, which are related to the two statements above, can be found in [13.

Example 3.11. Here is an example illustrating Theorem 3.3 . Let $B_{0}$ be a stationary simple Bratteli diagram with the matrix $A_{0}$ transpose to the incidence matrix of the diagram where

$$
A_{0}=\left(\begin{array}{ll}
1 & 1 \\
1 & 2
\end{array}\right) .
$$

Let $\leq_{0}$ be a proper order for $B_{0}$ and $\sigma$ be a substitution read on $\left(B_{0}, \leq_{0}\right)$. For example, choose

$$
\sigma=\left\{\begin{aligned}
a & \mapsto a b \\
b & \mapsto a b b .
\end{aligned}\right.
$$

Since substitution $\sigma$ is Sturmian, we have $p_{\sigma}(n)=n+1$ (see [9]). By Lemma 3.2. we build a primitive matrix $A_{1} \in \operatorname{Mat}(\mathbb{N}, 3)$ such that $S\left(A_{1}\right)=S(A)$ :

$$
A_{1}=\left(\begin{array}{ccc}
1 & 1 & 1 \\
2 & 3 & 1 \\
8 & 13 & 0
\end{array}\right) \text {. }
$$

Consider a proper order $\leq_{1}$ on $B_{1}$ with the following substitution $\zeta$ read on $\left(B_{1}, \leq_{1}\right)$ :

$$
\zeta=\left\{\begin{array}{l}
a \mapsto a b b c c c c c c c c, \\
b \mapsto a b b b c c c c c c c c c c c c, \\
c \mapsto a b .
\end{array}\right.
$$

It can be proved that $p_{\zeta}(n) \geq 3 n$. Hence we already obtain that $\left(X_{\sigma}, T_{\sigma}\right)$ and $\left(X_{\zeta}, T_{\zeta}\right)$ are non-isomorphic orbit equivalent systems.

Remark 3.12. Let $\lambda$ be the Perron-Frobenius eigenvalue of $A_{0}$ and $x$ be the PerronFrobenius eigenvector. Let $\mu$ be the unique $\mathcal{R}$-invariant measure for the corresponding diagram. Then $\lambda H(x)=H(x)$ and $G(S(\mu))=H(x)$ (see [2]).

Example 3.13. The following example concerns Lemma 3.5. Lind [13. pointed out an example of Perron number $\lambda(\lambda \approx 3.8916$ is the Perron root of the equation $f(t)=t^{3}+3 t^{2}-15 t-46$ and $\left.\operatorname{deg} \lambda=3\right)$ such that there exists no matrix $A \in$ $\operatorname{Mat}(\mathbb{N}, 3)$ with Perron-Frobenius eigenvalue $\lambda$. The reason is that a 3 -dimensional matrix with spectral radius $\lambda$ has trace -3 and hence cannot be non-negative. Lemma 3.5 states that such a matrix must exist for some power of $\lambda$. Here we 
present a matrix $\widetilde{A} \in \operatorname{Mat}(\mathbb{N}, 3)$ with Perron-Frobenius eigenvalue $\lambda^{M}$ for some $M \in \mathbb{N}$. In the notation used in Lemma 3.5, we notice that

$$
C=\left(\begin{array}{ccc}
0 & 0 & 46 \\
1 & 0 & 15 \\
0 & 1 & -3
\end{array}\right),
$$

and $\mathbf{y}_{1}=\left(1, \frac{1}{\lambda}+\frac{15}{46}, \frac{1}{\lambda^{2}}+\frac{15}{46} \frac{1}{\lambda}-\frac{3}{46}\right)^{T}$ has positive coordinates (see also [2]). Let $\left\{\mathbf{e}_{i}\right\}_{i=1}^{3}$ be the standard basis of $\mathbb{Q}^{3}$; hence $\left\langle\mathbf{e}_{i}, \mathbf{n}\right\rangle=\lambda^{i-1}>0$. Then the iterates of matrix $C$ drive each $\mathbf{e}_{i}$ closer to $\mathbf{y}_{1}$ when $M$ is growing; hence $C^{M} \mathbf{e}_{i}$ has all positive coordinates for sufficiently large $M$. We can choose $\widetilde{A}=C^{M}$. In this specific example, it suffices to take $M \geq 49$.

\section{ACKNOWLEDGEMENT}

The authors thank the referee for detailed comments on the paper, which helped to make the exposition much better.

\section{REFERENCES}

[1] Ethan Akin, Good measures on Cantor space, Trans. Amer. Math. Soc. 357 (2005), no. 7, 2681-2722 (electronic), DOI 10.1090/S0002-9947-04-03524-X. MR2139523(2006e:37003)

[2] S. Bezuglyi and O. Karpel, Homeomorphic measures on stationary Bratteli diagrams, J. Funct. Anal. 261 (2011), no. 12, 3519-3548, DOI 10.1016/j.jfa.2011.08.009. MR2838033 (2012i:37001)

[3] S. Bezuglyi, J. Kwiatkowski, and K. Medynets, Aperiodic substitution systems and their Bratteli diagrams, Ergodic Theory Dynam. Systems 29 (2009), no. 1, 37-72, DOI 10.1017/S0143385708000230. MR2470626 (2009m:37020)

[4] S. Bezuglyi, J. Kwiatkowski, K. Medynets, and B. Solomyak, Invariant measures on stationary Bratteli diagrams, Ergodic Theory Dynam. Systems 30 (2010), no. 4, 973-1007, DOI 10.1017/S0143385709000443. MR2669408 (2012g:37019)

[5] Julien Cassaigne, Special factors of sequences with linear subword complexity, Developments in language theory, II (Magdeburg, 1995), World Sci. Publ., River Edge, NJ, 1996, pp. 25-34. MR $1466182(98 \mathrm{e}: 68212)$

[6] Julien Cassaigne, Complexité et facteurs spéciaux (French, with English and French summaries), Journées Montoises (Mons, 1994). Bull. Belg. Math. Soc. Simon Stevin 4 (1997), no. 1, 67-88. MR.1440670 (98c:68176)

[7] F. Durand, B. Host, and C. Skau, Substitutional dynamical systems, Bratteli diagrams and dimension groups, Ergodic Theory Dynam. Systems 19 (1999), no. 4, 953-993, DOI 10.1017/S0143385799133947. MR1709427 (2000i:46062)

[8] Sébastien Ferenczi, Complexity of sequences and dynamical systems, Combinatorics and number theory (Tiruchirappalli, 1996). Discrete Math. 206 (1999), no. 1-3, 145-154, DOI 10.1016/S0012-365X(98)00400-2. MR 1665394 (2000f:68093)

[9] N. Pytheas Fogg, Substitutions in dynamics, arithmetics and combinatorics, edited by V. Berthé, S. Ferenczi, C. Mauduit and A. Siegel, Lecture Notes in Mathematics, vol. 1794, Springer-Verlag, Berlin, 2002. MR.1970385 (2004c:37005)

[10] A. H. Forrest, K-groups associated with substitution minimal systems, Israel J. Math. 98 (1997), 101-139, DOI 10.1007/BF02937330. MR1459849(99c:54056)

[11] Thierry Giordano, Ian F. Putnam, and Christian F. Skau, Topological orbit equivalence and $C^{*}$-crossed products, J. Reine Angew. Math. 469 (1995), 51-111. MR1363826 (97g:46085)

[12] Richard H. Herman, Ian F. Putnam, and Christian F. Skau, Ordered Bratteli diagrams, dimension groups and topological dynamics, Internat. J. Math. 3 (1992), no. 6, 827-864, DOI 10.1142/S0129167X92000382. MR.1194074 (94f:46096)

[13] D. A. Lind, The entropies of topological Markov shifts and a related class of algebraic integers, Ergodic Theory Dynam. Systems 4 (1984), no. 2, 283-300, DOI 10.1017/S0143385700002443. MR.766106 (86c:58092) 
[14] Konstantin Medynets, Cantor aperiodic systems and Bratteli diagrams (English, with English and French summaries), C. R. Math. Acad. Sci. Paris 342 (2006), no. 1, 43-46, DOI 10.1016/j.crma.2005.10.024. MR2193394 (2006g:37011)

[15] Melvyn B. Nathanson, Additive number theory, Inverse problems and the geometry of sumsets. Graduate Texts in Mathematics, vol. 165, Springer-Verlag, New York, 1996. MR.1477155 (98f:11011)

[16] Martine Queffélec, Substitution dynamical systems - spectral analysis, 2nd ed., Lecture Notes in Mathematics, vol. 1294, Springer-Verlag, Berlin, 2010. MR2590264 (2011b:37018)

[17] Hisatoshi Yuasa, On the topological orbit equivalence in a class of substitution minimal systems, Tokyo J. Math. 25 (2002), no. 2, 221-240, DOI 10.3836/tjm/1244208850. MR.1948661 (2003k:37024)

Institute for Low Temperature Physics, Kharkov, Ukraine

E-mail address: bezuglyi@ilt.kharkov.ua

Institute for Low Temperature Physics, Kharkov, Ukraine

E-mail address: helen.karpel@gmail.com 